\title{
Cyclobutadiene: The Antiaromatic Paradigm?
}

\author{
Thomas Bally*
}

\author{
Keywords: \\ antiaromaticity $\cdot$ cyclobutadiene $\cdot$ thermochemistry
}

$T_{\text {here }}$ molecule that has fascinated experimental and theoretical chemists so consistently over the past 40 years as has cyclobutadiene (CBD). On average, 16 publications which deal, in one form or another, with the parent compound $\mathrm{C}_{4} \mathrm{H}_{4}$ and 40 which deal with cyclobutdienes in general have appeared in every year of this time span, and there are probably many more on derivatives and metal complexes. There is no sign that interest in CBD is about to wane, as the highest number of papers per year appeared in 2000 and 2002, and in the last five years two entire thematic issues of Chemical Reviews, both with many references to $\mathrm{CBD}$, were dedicated to issues of aromaticity/antiaromaticity ${ }^{[1]}$ and localization/delocalization, ${ }^{[2]}$ that is, topics that are central to the debate on CBD.

Recently the first direct and reasonably accurate determination of the enthalpy of formation of CBD has been achieved by the group of Kass. ${ }^{[3,4]}$ Thus they have provided a solid foundation for the discussion on the thermochemical consequences of antiaromaticity, and I take this occasion to reflect on recent developments in this field and to point out the pitfalls with which the resulting discussion is fraught.

In spite of the great activity documented in the opening paragraph, many myths surround this fascinating molecule, most of which relate to the extent of delocalization of its $\pi$ electrons and/ or the degree of antiaromaticity. It is

[*] Prof. T. Bally

Chemistry Department

University of Fribourg

1700 Fribourg (Switzerland)

E-mail: thomas.bally@unifr.ch

Homepage:

http://www-chem.unifr.ch/tb/bally/ widely accepted (and hardly disputed) that benzene, with its six cyclically delocalized $\pi$ electrons, represents the paradigm of aromaticity. In view of the famous Hückel $4 n / 4 n+2$ electron rule, one is tempted to rush to the conclusion that $\mathrm{CBD}$, with its four cyclically delocalized $\pi$ electrons, is therefore the paradigm of antiaromaticity.

However, this conclusion is not as straightforward as it might seem, because in benzene the two Kekule resonance structures contribute with equal weights to the $D_{6 h}$ equilibrium geometry, whereas in CBD, with its rectangular structure having strongly localized single and double bonds, this is obviously not the case. Thus the notion of "bond delocalization" does not have the same meaning in the two compounds, and one of the issues of the continuing debate is to what extent this affects the properties that are associated with (anti)aromaticity. Another issue is of course how exactly one should or should not assess these properties. I will comment herein on some aspects of this debate.

Various criteria have been proposed over time to judge whether a molecule is aromatic or not (it is perhaps worth recalling that the term "aromatic" itself implies that a pleasant odor must at one time have been considered as an important criterion; thus, by the same token, antiaromatic compounds should smell unpleasantly), and it was generally assumed that several of these criteria have to be met for a molecule to qualify for this distinction.

Two types of characteristic traits of aromaticity have emerged as "beacon" features. The first relates to magnetic properties, in particular the anisotropy of the diamagnetic susceptibility and its exaltation, which distinguishes aromatic compounds from polyenes. Following suggestions by Pauling and Londsdale,
In memory of Satoru Masamune (1928-2003)

London proposed in 1937 a very appealing model that accounts for these effects in terms of ring currents that are induced by the external magnetic field in the system of cyclically delocalized $\pi$ electrons. ${ }^{[5]}$

There has been a vigorous debate in recent years about whether and to what extent these ring currents contribute to the deshielding or shielding of protons attached to aromatic or antiaromatic rings, respectively, ${ }^{[6-8]}$ as manifested in ${ }^{1} \mathrm{H}$ NMR spectra. At the focus of this debate stand the "nuclear independent chemical shifts" (NICS values) ${ }^{[9]}$ that can be computed at any point in the space surrounding a molecule. It has become clear that the original propos$\mathrm{al}^{[9]}$ to calculate NICS values at the center of (anti)aromatic rings does not provide a valid measure of currents induced in the cycle of $\pi$ electrons. However, it seems that the sign and magnitude of the $\pi$ component of the magnetic-shielding (or NICS) tensor at some distance (usually $1 \AA$ ) above the ring provides a good indication of the direction and size of the ring current, even in systems that have only $\sigma$ electrons. ${ }^{[10]}$

However, I will focus herein on the other class of "beacon" properties that are characterictic of (anti)aromaticity, namely thermochemical and/or kinetic ones (the two often being mixed up). Clearly, benzene enjoys a special thermodynamic stability which expresses itself, for example, in the fact that its (mono)hydrogenation is endothermic by $22 \mathrm{~kJ} \mathrm{~mol}^{-1}$ (Scheme 1), in contrast to that of hexatriene, which is exothermic by about $114 \mathrm{~kJ} \mathrm{~mol}^{-1}$ (the exact number depends on the choice of conformations). As there is no evident reason why one or the other of the conjugated dienes that result from these hydrogenations should suffer from any 


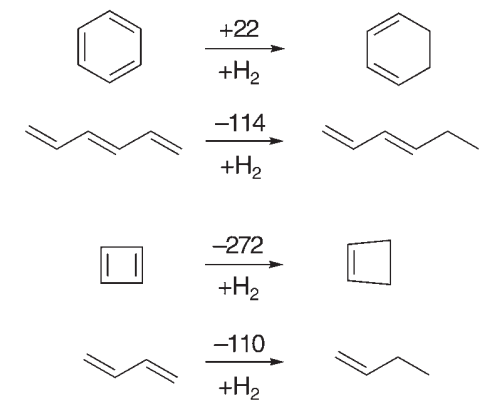

Scheme 1. Heats of hydrogenation of various compounds in $\mathrm{kJ} \mathrm{mol}^{-1}$. $^{[1]}$

special destabilization, and because both compounds may be regarded as essentially strain-free, the $136 \mathrm{~kJ} \mathrm{~mol}^{-1}$ difference must a result of some special stability of benzene compared to hexatriene. By the same token, the high exothermicity of the hydrogenation of CBD is an indication of a similar thermochemical destabilization of this compound (relative to butadiene, for example).

As long as the argument remains on such a semiquantitative level, there is no problem. However, if one wants to quantitate the "(anti)aromatic (de)stabilization energy" more precisely, one invariably hits upon the question of what exactly one should take as a reference, which could be either a real molecule or a theoretical construct. ${ }^{[12]}$ There is an enormous body of literature dealing with this very controversial question, literature which has been reviewed elsewhere. ${ }^{[13]}$ However, an important point is that one should carefully distinguish between the total energy that is gained by putting double bonds into (linear or cyclic) conjugation and the energy gained (or lost) exclusively by virtue of the conjugation being cyclic. The difference of these two energies may be termed "conjugation energy" because it accounts for conjugation, but not for cyclic resonance.

The total energy gain corresponds to the original Pauling-Wheland resonance energy, ${ }^{[14]}$ and it is invariably negative (that is, the energy is lowered), irrespective of whether the species under consideration is aromatic, nonaromatic, or antiaromatic. The second, which may be negative or positive, is usually termed Dewar resonance energy. ${ }^{[15,16]}$ It derives from the observation that enthalpies of atomization or for- mation of polyenes can be composed from experimentally based bond ${ }^{[15]}$ or group increments. ${ }^{[17]}$ Such increments allow one to calculate the energy of a (purely hypothetical) cyclic polyene that benefits from all the conjugation energy but not the special contribution resulting from the cyclic arrangements of double bonds that allows for equivalent resonance structures in the case of benzene.

The extra energy gained or lost in this way (that is, the Dewar resonance energy) is then equated with the difference between the experimental enthalpy of atomization or formation of the compound under scrutiny and its hypothetical polyene reference. If one uses the latest version of Benson-type group increments, ${ }^{[18]}$ this energy difference amounts to $87 \mathrm{~kJ} \mathrm{~mol}^{-1}$ in benzene. I believe that this procedure and the resulting Dewar resonance energy represent the best and most transparent way to assess the aromatic resonance stabilization in benzoid hydrocarbons. The results are indeed in good accord with determinations of the same quantity-usually by much more complicated procedures-relative to comparable (experimental or theoretical) standards.

In the case of benzene (or benzenoid hydrocarbons) the assessment of the Dewar resonance energy is greatly facilitated by the fact that this is the only nonadditive effect that needs to be considered, because such compounds are assumed to be free of strain (all bond angles have the ideal value of $120^{\circ}$ for $\mathrm{sp}^{2}$-hybridized carbon atoms, and the separations between nonbonding hydrogen atoms are larger than the sum of their van der Waals radii).

However, this is clearly not the case in CBD. If one compares the recently determined heat of formation of CBD $\left(429 \pm 16 \mathrm{~kJ} \mathrm{~mol}^{-1}\right)^{[3]}$ with that obtained by adding four polyenic $\mathrm{C}_{\mathrm{d}^{-}}(\mathrm{H})\left(\mathrm{C}_{\mathrm{d}}\right)$ group increments $\left(113 \mathrm{~kJ} \mathrm{~mol}^{-1}\right),{ }^{[18]}$ one is left with a whopping destabilization energy of $316 \pm 16 \mathrm{~kJ} \mathrm{~mol}^{-1}$. One then faces the problem of how to figure out which part of this energy results from some form of strain, and which results from antiaromatic destabilization.

It is very important to recognize (as Kass and co-workers did) that there is no unambiguous way to dissect the above number. If one wants to quantitate the antiaromatic destabilization energy of
$\mathrm{CBD}$, one must make some assumption about its strain energy. The most reasonable such assumption would seem to be that the strain energy increases linearly in the series cyclobutane/cyclobutene/CBD. In this case the antiaromatic destabilization energy is given by the enthalpy change for the isodesmic Reaction (1). ${ }^{[3]}$

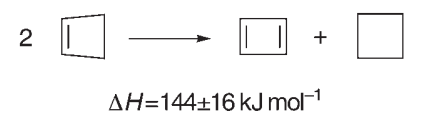

However, the above assumption merits some consideration. The fact that the strain energy increases by a mere $4 \mathrm{~kJ} \mathrm{~mol}^{-1}$ on going from cyclobutane to cyclopropane indicates that the energetic cost of angular deformation (Baeyer strain) cannot account for this property alone. Two different explanations have been advanced to account for this discrepancy. The first argues that the increase in angular strain between the two compounds is largely balanced by an increase in the strength of the $\mathrm{C}-\mathrm{H}$ bonds, owing to rehybridization of the ring carbon atoms, and that the strain energy of cyclopropane is therefore anomalously low, whereas that of cyclobutane is rather normal. ${ }^{[19]}$ The second explanation refers to the proposal, originally advanced by Dewar, ${ }^{[20]}$ that cyclic delocalization of $4 n+2$ or $4 n \sigma$ electrons in $\mathrm{C}-\mathrm{C}$ bonds may also lead to aromatic stabilization or destabilization, respectively. ${ }^{[10]}$ Accordingly, cyclopropane is stabilized by this so-called $\sigma$ aromaticity (which manifests itself, for example, in negative NICS values above the ring plane), whereas cyclobutane is destabilized by $\sigma$ antiaromaticity (positive NICS values). ${ }^{[10]}$ Although the energetic consequences of this effect cannot be directly assessed, they have been proposed to account for the surprisingly small difference in strain energies between cyclopropane and cyclobutane. Perhaps both of the above explanations need to be invoked to arrive at a complete picture of "strain" in the two compounds.

An additional complication, pointed out originally by Politzer et al., ${ }^{[21]}$ concerns the observation that in CBD two $\pi$ bonds are forced into a much more proximate position than in any other polyene. The fact that the $\mathrm{C}-\mathrm{C}$ bond 
length in CBD (ca. $1.57 \AA$ according to high-level calculations ${ }^{[22]}$ ) is nearly $0.1 \AA$ longer than in butadiene, whereas the $\mathrm{C}=\mathrm{C}$ bond length is similar in the two compounds, may be an expression of a substantial repulsion between the two $\pi$ bonds. However, it is difficult to quantitate this repulsion, and the pronounced alternation in bond length in CBD may also be attributed to strong vibronic coupling between the ground electronic state and the first excited singlet state. ${ }^{[23]}$

Two corollaries follow from the above considerations: a) "strain" is a multifaceted property which is composed of several nonadditive effects that were invented by chemists to understand this property, and it cannot be reduced to angular deformation and/or steric repulsion; b) it is therefore not a priori clear why and how this property changes on going from cyclobutane to cyclobutene to CBD. It is unclear whether $\sigma$ antiaromaticity is the same for puckered (as in cyclobutane) and planar (as in CBD) four-membered rings and how it depends on the size of the ring. If $\sigma$ antiaromaticity does contribute significantly to the destabilization of CBD, the tantalizing question arises as to whether this should be counted towards antiaromaticity or towards strain.

In view of all the above it is not surprising that recent opinions vary enormously on how much CBD is thermochemically destabilized solely by virtue of the cyclic conjugation of its four $\pi$ electrons; estimations range from $230 \mathrm{~kJ} \mathrm{~mol}^{-1} \quad$ (Deniz et al. ${ }^{[24]}$ to $44 \mathrm{~kJ} \mathrm{~mol}^{-1}$ (Mo and Schleyer ${ }^{[13]}$ ). If even this lower number is attributed to $\pi$ repulsion, one is left with the prediction that $\mathrm{CBD}$, in spite of its enormous destabilization, must be considered as essentially nonaromatic, as based on its thermochemical properties. In fact, Schleyer concludes in his above-cited paper $^{[13]}$ that "instead of the conventional interpretation of $\mathrm{CBD}$ as the antiaromatic paradigm, it should be regarded as a unique molecule", a view that I tend to share.

What other properties might be associated with antiaromaticity in CBD? The ionization energy of CBD $\left(8.16 \mathrm{eV}^{[25]}\right)$ is nearly $1 \mathrm{eV}$ lower than that of butadiene $(9.08 \mathrm{eV})$, whereas that of benzene $(9.25 \mathrm{eV})$ is higher than that of hexatriene $(8.29 \mathrm{eV})$ by a similar amount. What this comparison indicates is that the energy of the $\pi$ HOMO of CBD is raised by cyclic conjugation, while it is lowered in benzene. Of course this trend carries over to the total $\pi$ energy, which in the case of CBD is the same as that of two ethylene molecules $(4 \alpha+4 \beta$ in Hückel molecular orbital terms) and nearly $0.5 \beta$ higher than that of butadiene, whereas the total $\pi$ energy of benzene is lower than that of three ethylene molecules by $2 \beta$ (and lower than that of hexatriene by about $1 \beta)$. In this perspective the ionization potential is an expression of (anti)aromaticity.

As CBD and benzene are alternant hydrocarbons, their HOMO-LUMO gaps should correlate with the HOMO energy (that is, the ionization potential). For that reason, the energies of the lowest singlet states should also be very different in the two compounds. Unfortunately, this comparison is hampered by the fact that, owing to the degeneracies of the frontier MOs, the lowest excited singlet state of benzene cannot be described by simple HOMOLUMO excitation. ${ }^{[26]}$ However, the real problem is that the absorption spectrum of CBD has not been unambiguously assessed by experiment. An absorption band observed at $300 \mathrm{~nm}^{[27]}$ has never been confirmed as such, but cleavage of CBD upon irradiation at $350-420 \mathrm{~nm}$ to give acetylene ${ }^{[28]}$ indicates that $\mathrm{CBD}$ must absorb in this region, albeit too weakly for a band to be observed.

Thus, we must rely on excited-state calculations, which are surprisingly scarce. $^{[29]}$ According to these calculations, rectangular CBD has two nearUV electric-dipole-forbidden transitions that correspond to the excitation of one $\left(1^{1} A_{g} \rightarrow 1^{1} B_{1 g}\right)$ or two electrons $\left(1^{1} A_{g} \rightarrow\right.$ $2^{1} A_{g}$ ) from the HOMO to the LUMO. The recent coupled-cluster calculations of Levchenko and Krylov ${ }^{[22]}$ predict that these two transitions occur (vertically) at 360 and $285 \mathrm{~nm}$. According to our own CASPT2 calculations, ${ }^{[30]}$ the first allowed transition $\left(1^{1} \mathrm{~A}_{\mathrm{g}} \rightarrow 1^{1} \mathrm{~B}_{3 \mathrm{u}}\right)$ occurs above $7 \mathrm{eV}(<180 \mathrm{~nm})$, that is, in the vacuum-UV region. Thus, the observed near-UV photocleavage of CBD must indeed involve HOMO-LUMO excitation at around $1.5 \mathrm{eV}$ lower in energy than in benzene.
Finally there is the question of the rate and automerization barrier of the two rectangular structures of CBD. Experiment has so far only provided rough estimates and limits. From trapping studies in solution, Whitman and Carpenter concluded that the barrier must lie between 6.7 and ca $40 \mathrm{~kJ} \mathrm{~mol}^{-1}$, 29$]$ while Orendt et al. deduced from IR and NMR measurements in cryogenic matrices that the rate constant of interconversion must be higher than $10^{3}$ at $25 \mathrm{~K},{ }^{[30]}$ which translates into a free energy of activation of less than $4 \mathrm{~kJ} \mathrm{~mol}^{-1}$. However, the best high-level $\mathrm{ab}$ initio calculations predict the barrier to lie between 27 and $32 \mathrm{~kJ} \mathrm{~mol}^{-1}$. ${ }^{[31,32]}$ These two facts can only be reconciled if one invokes heavy-atom tunnelling. In 1983 Carpenter had in fact done exactly that to account for the negative entropy of activation which he had deduced from his experiments, ${ }^{[2]}$ and he concluded that the tunnelling mechanism would actually dominate the automerization process at temperatures below $0{ }^{\circ} \mathrm{C}^{[33]}$ Although this proposal originally met with a great deal of scepticism, it has not been dispelled to date.

Thus, cyclobutadiene continues to fascinate chemists and it will certainly continue to serve as a benchmark and testing ground for different theoretical models that chemists come up with to make sense of the sometimes ambivalent manifestations of aromatic or antiaromatic molecules. Now that a wellfounded number is available for the heat of formation of $\mathrm{CBD}$, the purported paradigm of antiaromaticity, any speculation about the contributions of various effects to its destabilization rests at least on solid ground.

I am indebted to Profs. Barry Carpenter (Cornell University, now University of Cardiff) and Josef Michl (University of Colorado, Boulder) for critical reading of the manuscript.

[1] Chem. Rev. 2001, 101(5), 1115.

[2] Chem. Rev. 2005, 105(10), 3433.

[3] A. Fattahi, L. Liz, Z. Tian, S. R. Kass, Angew. Chem. 2006, 118, 5106; Angew. Chem. Int. Ed. 2006, 45, 4984.

[4] The enthalpy of formation of cyclobutadiene had been previously derived from 
the heat liberated during the photodecomposition of a polycyclic cyclobutadiene precursor (see reference [24]). However, owing to various assumptions that had to be made with regard to the thermochemical properties of this precursor and side products of this reaction, their resulting estimate of $\Delta_{\mathrm{f}} H^{\circ}(\mathrm{CBD})=$ $477 \pm 46 \mathrm{~kJ} \mathrm{~mol}^{-1}$ turned out to be significantly too high.

[5] F. C. London, J. Chem. Phys. 1937, 5, 837.

[6] C. S. Wannere, P. von R. Schleyer, Org. Lett. 2003, 5, 605.

[7] R. Viglione, R. Zanasi, P. Lazzeretti, Org. Lett. 2004, 6, 2265

[8] A. Stanger, J. Org. Chem. 2005, 70, 883.

[9] P. von R. Schleyer, C. Maerker, A. Dransfeld, H. Jiao, N. J. R. van Eikema Hommes, J. Am. Chem. Soc. 1996, 118, 6317.

[10] D. Moran, M. Manoharan, T. Heine, P. von R. Schleyer, Org. Lett. 2003, 5, 23.

[11] Based on enthalpies of formation from: H. Y. Afeefy, J. F. Liebman, S. E. Stein in NIST Chemistry WebBook, NIST Standard Reference Database No 69 (Eds.: P. J. Lonstrom, W. G. Mallard), National Institute of Standards and Technology, Gaithersburg, MD, 2005 (http://webbook.nist.gov/chemistry), and the enthalpy of formation of cyclobutadiene from the recent work of Kass and co-workers. ${ }^{[3]}$

[12] Notwithstanding repeated and continuing claims to the contrary, especially by theoreticians, it is impossible to measure (anti)aromaticity "absolutely", that is, without recourse to a nonaromatic reference or standard.

[13] Y. Mo, P. von R. Schleyer, Chem. Eur. J. 2006, 12, 2009 and references cited therein.

[14] L. C. Pauling, G. W. Wheland, J. Chem. Phys. 1933, 1, 362.

[15] M. J. S. Dewar, C. de Llano, J. Am. Chem. Soc. 1969, 91, 789.

[16] L. J. Schaad, B. A. Hess, Chem. Rev. 2001, 101, 1465.

[17] S. W. Benson, Thermochemical Kinetics, Wiley, New York, 1976.

[18] E. S. Domalski, E. S. Hearing, J. Phys. Chem. Ref. Data 1993, 22, 1963.

[19] R. D. Bach, O. Dmitrenko, J. Am. Chem. Soc. 2004, 126, 4444. According to calculations, the strength of the $\mathrm{C}-\mathrm{H}$ bonds (that is, their dissociation enthalpy) increases by about $35 \mathrm{~kJ} \mathrm{~mol}^{-1}$ on going from cyclobutane to cyclopropane. In order to keep the overall strain energies of the two compounds as similar as they are, the increase in angular strain on narrowing the C-C-C bond angles from $90^{\circ}$ to $60^{\circ}$ would therefore have to be about $70 \mathrm{~kJ} \mathrm{~mol}^{-1}$ per $\mathrm{CH}_{2}$ group, that is, the angular strain would have to be over $200 \mathrm{~kJ} \mathrm{~mol}^{-1}$ higher in cyclopropane than in cyclobutane! This shows once more that dissecting nonadditive effects is a delicate matter.

[20] M. J. S. Dewar, J. Am. Chem. Soc. 1984, 106, 669.
[21] P. Politzer, M. E. Grice, J. S. Murray, J. M. Seminario, Can. J. Chem. 1993, 71, 1123.

[22] S. V. Levchenko, A. I. Krylov, J. Chem. Phys. 2004, 120, 175.

[23] S. Zilberg, Y. Haas, J. Phys. Chem. A 1998, 102, 10843.

[24] A. A. Deniz, K. S. Peters, G. J. Snyder, Science 1999, 286, 1119.

[25] D. W. Kohn, P. Chen, J. Am. Chem. Soc. 1993, 115, 2844.

[26] L. Salem, Molecular Orbital Theory of Conjugated Systems, W. Benjamin, New York, 1966, chap. 4.

[27] T. Bally, S. Masamune, Tetrahedron 1980, 36, 343.

[28] B. R. Arnold, J. Michl, J. Phys. Chem. 1993, 97, 13348.

[29] B. R. Arnold, J. Michl, in Kinetics and Spectroscopy of Carbenes and Biradicals (Ed.: M. S. Platz), Plenum Press, New York, 1990, p. 20.

[30] H. Aboulfadl, T. Bally, unpublished results.

[31] D. W. Whitman, B. Carpenter, J. Am. Chem. Soc. 1982, 104, 6473.

[32] A. M. Orendt, B. R. Arnold, J. G. Radziszewski, J. C. Facelli, K. D. Malsch, H. Strub, D. M. Grant, J. Michl, J. Am. Chem. Soc. 1988, 110, 2648.

[33] A. Balkova, R. J. Bartlett, J. Chem. Phys. 1994, 101, 8972.

[34] S. V. Levchenko, T. Wang, A. I. Krylov, J. Chem. Phys. 2005, 122, 224106.

[35] B. Carpenter, J. Am. Chem. Soc. 1983, 105, 1700. 